\title{
Grupo Drosophila asiri (Diptera, Drosophilidae), un nuevo grupo de especies andinas con la descripción de dos nuevas especies y la redescripción de Drosophila asiri
}

\author{
María Luna Figuero, Violeta Rafael \& Diego Céspedes
}

Laboratorio de Genética Evolutiva, Escuela de Ciencias Biológicas, Pontificia Universidad Católica del Ecuador, 17-01-2184, Quito, Ecuador. (lunaf3@hotmail.com; vrafael@puce.edu.ec; diego_cesp@hotmail.com)

\begin{abstract}
Drosophila asiri group (Diptera, Drosophilidae), a new group of Andean species with the description of two new species and the redescription of Drosophila asiri. The Drosophila asiri group is a new group of species in the subgenus Drosophila. The specimens were captured in the highlands of Ecuador from $3200 \mathrm{~m}$ to $4000 \mathrm{~m}$ above sea level. This group includes Drosophila (D.) asiri Vela \& Rafael, 2005, that was previously described within the D. onychophora species group, and two new species, Drosophila (D.) yuragyacum sp. nov. and Drosophila (D.) yanaurcus sp. nov. These species were captured using banana and yeast bait, in bosque protector Pasochoa, in the quebrada de Cruz Loma and in páramo de Papallacta. The members of the D. asiri species group appear to be endemic to the Ecuadorian Andes. All three species of the D. asiri group are large flies (about $6 \mathrm{~mm}$ ). Males have a sclerotized aedeagus with two well sclerotized lateral projections in the shape of horns. These characteristics distinguish members of this species group from other groups within Drosophila.
\end{abstract}

KEYWORDS. Highlands, Ecuador, endemism, Neotropical.

RESUMEN. El nuevo grupo Drosophila asiri pertenece al subgénero Drosophila. Los ejemplares fueron capturados en los bosques andinos del Ecuador, desde los 3200 hasta los $4000 \mathrm{~m}$ de altitud. A este grupo pertenecen Drosophila (D.) asiri Vela \& Rafael, 2005 previamente ubicada dentro del grupo D. onychophora, y dos nuevas especies; Drosophila (D.) yuragyacum sp. nov. y Drosophila (D.) yanaurcus sp. nov. Las capturas se realizaron en el bosque protector Pasochoa, en la quebrada de Cruz Loma y en el páramo de Papallacta, utilizando cebo de banano y levadura. Los miembros del nuevo grupo por el momento son endémicos de los Andes ecuatorianos. Las tres especies del grupo $D$. asiri son moscas de tamaño grande (aproximadamente $6 \mathrm{~mm}$ ). Los machos presentan el edeago quitinizado con dos proyecciones laterales en la cabeza a manera de cuernos muy quitinizados. Estas características diferencian a las especies del grupo D. asiri de otros grupos dentro de Drosophila.

PALABRAS-CLAVE. Bosques andinos, Ecuador, endemismo, Neotropical.

Vela \& Rafael (2005) describieron una nueva especie del género Drosophila capturada en el bosque protector Pasochoa (Pichincha, Ecuador); denominada como Drosophila asiri y fue ubicada dentro del grupo $D$. onychophora debido a las similitudes a nivel de la genitalia de los machos. La hembra no pudo ser identificada.

En el 2008 y 2009, Céspedes y Figuero respectivamente, recolectaron dos especies nuevas similares a $D$. asiri en su morfología externa e interna. La primera especie fue capturada en la quebrada de Cruz Loma, un bosque andino ubicado a $3500 \mathrm{~m}$ de altitud y la segunda en el páramo de Papallacta en un bosque de Polylepis (Rosaceae) a $4000 \mathrm{~m}$ de altitud. En ambas especies se pudo identificar a las hembras, lo cual permitió constatar que $D$. asiri no pertenecía al grupo $D$. onychophora, ya que los ovipositores de las otras dos especies eran diferentes a los ovipositores de las hembras del grupo D. onychophora (Vilela \& Bächli, 1990).

Estas especies presentan en la genitalia características que no comparten con ningún otro grupo de especies de Drosophila. Por ello se reúne a las tres especies en un nuevo grupo denominado grupo Drosophila asiri por ser el nombre de la primera especie descrita.

\section{MATERIALES Y MÉTODOS}

Se realizaron recolecciones de drosófilas en varias localidades de la provincia de Pichincha. Para las capturas se utilizó cebo de banano y levadura colocado en el interior de una botella plástica con pequeños agujeros. En Cruz Loma y Papallacta se colocaron data loggers en los transectos para tomar datos de humedad relativa y temperatura. Los individuos recolectados fueron llevados al laboratorio; con las hembras vivas se fundaron isolíneas en medio de cultivo de banano gelatina (RAfAel et al., 2000) y uvilla (Physalis peruviana L.) fresca. La identificación de las especies fue realizada mediante el análisis de la morfología externa y de la genitalia. Se utilizó Bächlı et al. (2004) como referencia para nombrar las estructuras en las descripciones de las especies, así como para los índices alares.

Los holotipos y paratipos de las nuevas especies están depositados en el Museo de Zoología, sección Invertebrados de la Pontificia Universidad Católica del Ecuador, Quito (QCAZ-I).

\section{RESULTADOS}

\section{Grupo de especies Drosophila asiri (grupo nuevo)}

Diagnosis. Longitud total $6 \mathrm{~mm}$ promedio. Tórax con una franja longitudinal más oscura. Abdomen con un diseño en la parte central de cada tergito en forma rectangular. Edeago quitinizado, con dos proyecciones laterales a manera de cuernos.

Morfología externa macho y hembra. Las especies de este grupo son moscas grandes de aproximadamente $6 \mathrm{~mm}$ de longitud total (cuerpo + alas). El cuerpo mide 
aproximadamente $4 \mathrm{~mm}$ y son moscas de color marrón amarillento o marrón. Carina prominente y surcada. En la parte media del tórax presentan una franja longitudinal más oscura; sin cerdas prescutelares. Las alas miden de 4,1 a $4,8 \mathrm{~mm}$ con la vena transversal posterior sombreada. En la parte media de cada tergito del abdomen presenta una zona oscura de forma rectangular (trapecio) y en la parte inferior presenta una línea delgada oscura.

Genitalia externa macho. La placa anal es micropubescente con cerdas largas y está parcialmente fusionada al epandrio (arco genital). Surestilo con dientes primarios que varían de 9 a 18 (a veces separados), sin dientes secundarios; con 12 a 23 cerdas marginales y micropubescente en la parte externa de los dientes primarios. Epandrio micropubescente con cerdas; la parte baja del epandrio es desnuda y termina en una punta quitinizada.

Genitalia interna macho. Edeago quitinizado, con dos proyecciones laterales a manera de cuernos muy quitinizados. Cabeza del edeago ancha y aserrada lateralmente.

Genitalia hembra. Ovipositor quitinizado, de color marrón amarillento con una espina larga y de dos a tres espinas cortas. Espermateca pequeña muy quitinizada, redonda y con una invaginación que alcanza casi todo el largo de la espermateca.

El grupo $D$. asiri pertenece al subgénero Drosophila. Las especies de este nuevo grupo han sido encontradas en los bosques andinos del Ecuador desde los 3260 hasta los $4014 \mathrm{~m}$ de altitud.

\section{Drosophila asiri Vela \& Rafael, 2005}

$$
\text { (Figs 1-5, 21) }
$$

Material tipo. Holótipo $\widehat{o}$ (disectado, genitalia en microtubo, montado en seco), Ecuador, Pichincha, Volcán Pasochoa (Refugio de Vida Silvestre Pasochoa), VI.1996, D. Vela col., D. Vela det. (QCAZI 1704); paratipo $\hat{o}$ (disectado, genitalia en microtubo, montado en seco), los mismos datos del holotipo, 20.X.2001, V. Rafael \& D. Vela

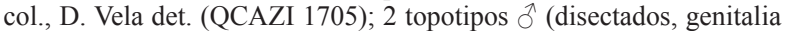
en microtubo, montados en seco), IV, VII.2001, V. Rafael \& D. Vela col., D. Vela det. (depositados en la colección del Laboratorio de Genética Evolutiva, PUCE); $\widehat{\partial}$ (disectado, genitalia en microtubo, montado en seco), Quito (Cruz Loma, 0 ${ }^{\circ} 1^{\prime} 19^{\prime \prime}$ 'S, 78 $31^{\prime} 25.1$ 'W, 3325 m), X.2008, D. Céspedes col., D. Céspedes det. (depositado en la colección del laboratorio de Genética Evolutiva, PUCE).

Diagnosis. Tórax con una franja longitudinal, oscura que abarca seis hileras de pelos acrosticales. Abdomen con un patrón de pigmentación en forma de rectángulo central en cada tergito. Cabeza del edeago quitinizada, ancha y aserrada lateralmente. Parte ventral de la cabeza con dos proyecciones laterales como cuernos muy quitinizados.

Descripción del macho, holotipo, paratipo $\mathrm{y}$ topotipos (individuos en alcohol). Morfología externa: longitud total (cuerpo + alas) del holotipo 6,1 $\mathrm{mm}$, longitud total (cuerpo + alas) de los paratipos y topotipos 5,2-7 mm. Longitud del cuerpo del holotipo $3,3 \mathrm{~mm}$. Color del cuerpo marrón.
Cabeza. Arista plumosa presenta cuatro ramas dorsales y dos ramas ventrales más la terminal bifurcada. Cabeza de color marrón oscuro polinoso. Placa orbital marrón; con cerda orbital media $2 / 3$ de la anterior y más cerca al borde ocular. Triángulo ocelar marrón oscuro; ocelos de color amarillo. Triángulo frontal marrón, frontal vitta ligeramente anaranjado. Una cerda oral prominente. Carina prominente y surcada. Ojos de color marrón oscuro rojizo.

Tórax. Color marrón oscuro polinoso. Presenta seis hileras de pelos acrosticales entre las dorsocentrales anteriores. En la parte central presenta una franja oscura longitudinal que se extiende entre las cerdas dorsocentrales abarcando a seis hileras de pelos acrosticales. Escutelo del mismo color que el tórax con cerdas escutelares anteriores paralelas. Cerda esternopleural media 1/4 de la anterior. Patas de color amarillo.

Alas. Color marrón claro, ligeramente sombreadas en la celda marginal, submarginal y en la vena transversal posterior. La vena costal III presenta siete cerdas gruesas. Longitud del ala 4,8 $\mathrm{mm}$; índices alares: alar $=2,39 ; \mathrm{C}=4,7 ; \mathrm{ac}=1,66 ; \mathrm{hb}=0,17 ; 4 \mathrm{c}=0,54$; $4 v=1,48 ; 5 x=1,36 ; M=0,4$ y Prox $x=0,48$.

Abdomen. Color marrón amarillento, sin línea media dorsal; patrón de pigmentación en forma de rectángulo central en cada tergito, los límites inferiores de cada tergito oscurecidos, las zonas laterales de cada tergito claras.

Genitalia externa. Placa anal micropubescente, con cerdas largas y parcialmente fusionada al epandrio (arco genital). Epandrio micropubescente; parte baja del epandrio sin microproyecciones, quitinizada y en punta. Surestilos micropubescentes en la cara externa; con 12 dientes primarios (en otros individuos de 9 a 18); de uno a cuatro dientes inferiores se encuentran separados del resto. Presentan 15 cerdas marginales (en otros individuos de 12 a 23) (Fig. 1).

Genitalia interna. Cabeza del edeago quitinizada, ancha y aserrada lateralmente; en vista lateral de forma globosa. En la parte ventral de la cabeza presenta dos proyecciones laterales como cuernos muy quitinizados que nacen en la parte más alta y están dirigidos hacia la parte anterior; en vista dorsal se observa una abertura desde el nacimiento de los cuernos hasta la unión con el pie del edeago; frontalmente presenta una estructura membranosa. Paráfisis pequeña de forma redonda con una cerda larga y dos espinas cortas, además presenta más de 20 microproyecciones en la parte ventral. Índice del edeago $=0,81$ (Figs 2-4, 21). Hipandrio más grande que el epandrio, en forma de escudo, abierto en la parte posterior, gonopodios con una cerda (Fig. 5).

Descripción de la hembra. Se desconoce.

Etimología. Asiri palabra del idioma quichua que significa sonrisa. 

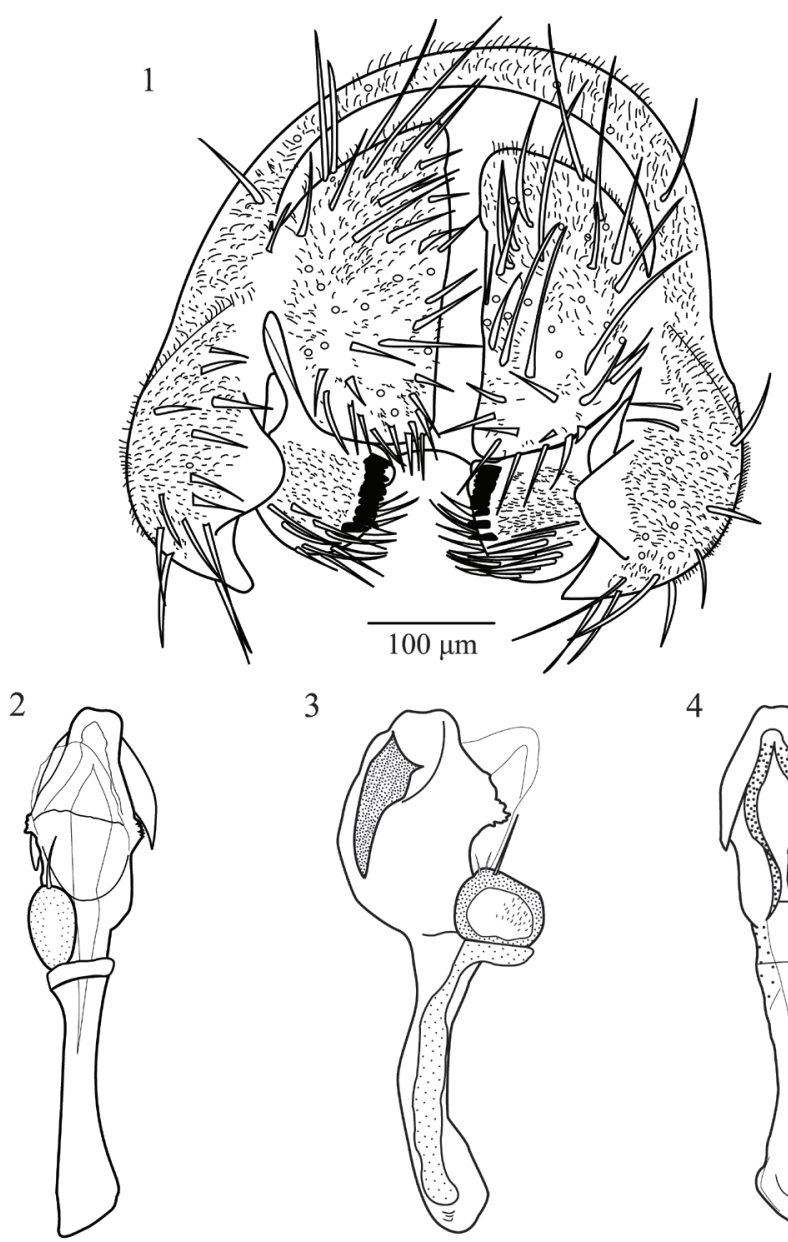

3

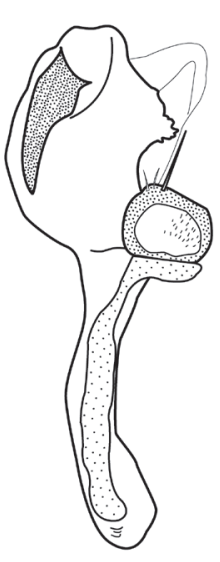

$100 \mu \mathrm{m}$

5

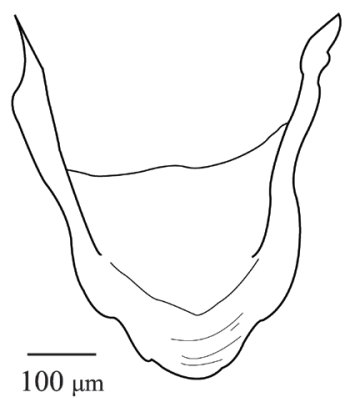

4

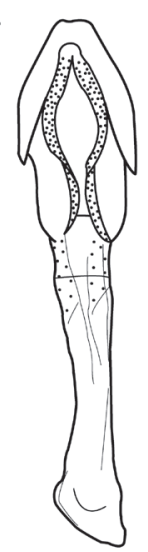

$100 \mu \mathrm{m}$

Figs 1-5. Drosophila (D.) asiri Vela \& Rafael, 2005, holotipo đ. 1, placa anal; edeago: 2, vista ventral; 3, vista lateral; 4, vista dorsal; 5, hipandrio.

\section{Drosophila (Drosophila) yuragyacum sp. nov.}

(Figs 6-12, 22, 24)

Material tipo. Holotipo $\hat{o}$ (disectado, genitalia en microtubo, montado en seco), Ecuador, Pichincha, Quito (Cruz Loma, $0^{\circ} 11$ '14,9'S, 78³1’38,9’W, 3.550m), VII.2008, D. Céspedes col., D. Céspedes det. (QCAZI 2292); alotipo 9 (disectado, genitalia en microtubo, montado en seco), los mismos datos del holotipo, D. Céspedes det. (QCAZI 2288); paratipo (disectado, genitalia en microtubo, montado en seco), los mismos datos del holotipo, D. Céspedes det. (QCAZI 2291).

Diagnosis. Tórax con una franja longitudinal oscura que abarca cinco hileras de pelos acrosticales. Abdomen con diseño oscuro en la parte central de cada tergito en forma de trapecio. Edeago quitinizado con dos proyecciones dorso-laterales a manera de cuernos y una proyección laminar. Ovipositor con cinco dientes discales, 25 cerdas marginales, una espina larga y dos cortas. Espermateca quitinizada con una invaginación que alcanza 3/4 de la espermateca.

Descripción del macho, holotipo y paratipo (individuos en alcohol). Morfología externa: longitud total (cuerpo + alas) del holotipo $6 \mathrm{~mm}$, longitud total (cuerpo + alas) del paratipo $5 \mathrm{~mm}$. Longitud del cuerpo del holotipo $4 \mathrm{~mm}$. Color del cuerpo marrón oscuro.

Cabeza. Primer y segundo segmento de las antenas de color amarillo, pero la base de estos segmentos de color más oscuro; arista plumosa, presenta cinco ramas 
dorsales y dos ventrales más la terminal bifurcada. Carina amarilla prominente, ligeramente surcada y tiene una banda marrón en la zona externa. Triángulo ocelar de color marrón oscuro, el frontal vitta amarillo pero la zona anterior es de color marrón oscuro. La cerda orbital media más cerca al borde del ojo y a la cerda orbital anterior. Tiene una cerda oral prominente. Los palpos son de color amarillo.

Tórax. De color marrón oscuro con siete hileras de pelos acrosticales entre las dorsocentrales anteriores. Cerdas escutelares anteriores divergentes. Presenta una franja longitudinal más oscura que se extiende entre las cerdas dorsocentrales abarcando a cinco hileras de los pelos acrosticales y que llega hasta el escutelo. A continuación hay una franja de color marrón claro en el punto donde emergen las cerdas dorsocentrales. En el borde interno de la cerda dorsocentral anterior hay una franja amarilla que se dirige hacia la parte anterior del tórax y luego se bifurca a lo largo del borde externo de las dorsocentrales. Cerda esternopleural media es 1/4 de la cerda esternopleural anterior. Patas de color amarillo.

Alas. Color marrón claro con la vena transversal posterior ligeramente sombreada. Longitud del ala 4,11 $\mathrm{mm}$, ancho del ala $1,78 \mathrm{~mm}$. Índices alares: $\mathrm{C}=6,91 ; \mathrm{ac}=$ 1,$5 ; \mathrm{hb}=0,33 ; 4 \mathrm{c}=0,34 ; 4 \mathrm{v}=1,37 ; 5 \mathrm{x}=0,83 ; \mathrm{M}=0,29$; Prox $\mathrm{x}=0,49$.

Abdomen. Presenta diseño oscuro en la parte central de cada tergito en forma de trapecio que se va difuminando hacia los extremos.

Genitalia externa. Placa anal micropubescente con cerdas y parcialmente fusionada al epandrio (arco genital). Epandrio micropubescente; parte baja del epandrio sin micro proyecciones, quitinizada y en punta. Surestilos rectangulares, micropubescentes en la cara externa; con 13 dientes primarios en el lado derecho y 12 en el izquierdo. Las cerdas marginales son 14 en ambos lados (Fig. 6).

Genitalia interna. El edeago es quitinizado con dos proyecciones dorso-laterales a manera de cuernos muy quitinizados. La base de la cabeza del edeago es quitinizada y presenta una proyección laminar (Fig. 9, pl) larga (más pequeña que en la especie seguiente), curva y quitinizada dirigida hacia la parte anterior. El extremo posterior de la cabeza del edeago es triangular con la punta cóncava agrietada en los márgenes laterales. En la parte más ancha de la cabeza del edeago presenta tres dientes. La parte distal del pie es membranoso. Presenta una rama ventral corta, paráfisis ovalada con una cerda larga. Índice del edeago= 1,09 (Figs 8-10, 22). Descripción de la hembra, alotipo (individuo en alcohol). Morfología externa. Igual que el macho.

Genitalia. Ovipositor quitinizado, de color amarillo; en el lado derecho posee cinco dientes discales, 25 cerdas marginales, una espina larga y dos cortas más delgadas; en el lado izquierdo presenta cinco dientes discales, 21 cerdas marginales, una espina larga y tres cortas más delgadas (Fig. 11). Espermateca pequeña muy quitinizada, redonda con una invaginación que alcanza $3 / 4$ de la espermateca. Índice de la espermateca $=0,8$ (Figs 12, 24).

Etimología. El nombre de la especie hace referencia a la loma Yuracyacu, lugar cercano a Cruz Loma, Pichincha, Ecuador. Palabras quichuas; yurag significa blanco y yacu, agua.

\section{Drosophila (Drosophila) yanaurcus sp. nov.}

(Figs 13- 20, 23, 25)

Material tipo. Holotipo $\widehat{\partial}$ (disectado, genitalia en microtubo, montado en seco), Ecuador, Pichincha, Papallacta (Páramo de Papallacta, $\left.0^{\circ} 19^{\prime} 24,8^{\prime \prime} \mathrm{S}, 78^{\circ} 12^{\prime} 42,8^{\prime \prime} \mathrm{W}, 4.005 \mathrm{~m}\right)$, II.2009, M. L. Figuero col., M. L. Figuero det. (QCAZI 2191); alotipo 9 (disectado, genitalia en microtubo, montado en seco), mismos datos del holotipo, IV.2009, M. L. Figuero col., M. L. Figuero det. (QCAZI 2202). Paratipos: $4 \hat{\jmath}$ (disectados, genitalia en microtubo, montados en seco), mismos datos del holotipo, M. L. Figuero det. (QCAZI 2195- 2198); $5 \hat{\jmath}, 7$ 우 (disectados, genitalia en microtubo, montados en seco), Páramo de Papallacta, 0²0’09,4”S, 78¹2'32,1”W, 4.014 m, II, IV, VII.2009, M. L. Figuero col., M. L. Figuero det. (QCAZI 2192-2194, 2200, 2201, 2203-2205, 2207, 2208, 2210, 2211); J, 3우 (disectados, genitalia en microtubo, montados en seco), Peñas Blancas, $0^{\circ} 19^{\prime} 13^{\prime \prime S}$, $78^{\circ} 13^{\prime} 28,1^{\prime \prime} W, 3.731$ m, II.2009, M. L. Figuero col., M. L. Figuero det. (QCAZI 2199, 2206, 2209, 2212).

Diagnosis. Tórax con una franja longitudinal oscura y delgada que abarca dos hileras de pelos acrosticales. Abdomen con el tercer y cuarto tergito más oscuros en la parte media con un diseño semejante a un reloj de arena. Cabeza del edeago quitinizada aserrada lateralmente con dos proyecciones dorso-laterales como cuernos; entre estos cuernos se observa digitaciones y una proyección laminar. Ovipositor con cuatro a cinco dientes discales, 17 a 27 cerdas marginales, una espina larga y de dos a tres espinas cortas. Espermateca quitinizada, redonda con una invaginación que alcanza $3 / 4$ de la espermateca.

Descripción del macho, holotipo y paratipos (individuos muertos en alcohol). Morfología externa: longitud total (cuerpo + alas) del holotipo $6 \mathrm{~mm}$, longitud total (cuerpo + alas) de los paratipos 7- 7,2 $\mathrm{mm}$. Longitud del cuerpo del holotipo $4 \mathrm{~mm}$. Color del cuerpo amarillo polinoso.

Cabeza. Primer y segundo segmento de las antenas color amarillo; arista plumosa, presenta cuatro ramas dorsales y tres ventrales (en otros individuos con cuatro ramas dorsales y dos ventrales), más la terminal bifurcada. Cabeza amarilla de aspecto polinoso. La placa orbital amarilla; la cerda orbital media, hacia el exterior de la placa orbital y ubicada más cerca de la cerda orbital anterior. Triángulo ocelar marrón, triángulo frontal de color amarillo polinoso, frontal vitta ligeramente anaranjado. Una cerda oral prominente. Carina prominente ligeramente surcada. Proboscis y palpos de color amarillo, palpos con tres cerdas (en otros individuos con dos cerdas). Ojos de color marrón rojizo.

Tórax. Color amarillo polinoso. Presenta seis hileras de pelos acrosticales entre las dorsocentrales 
6

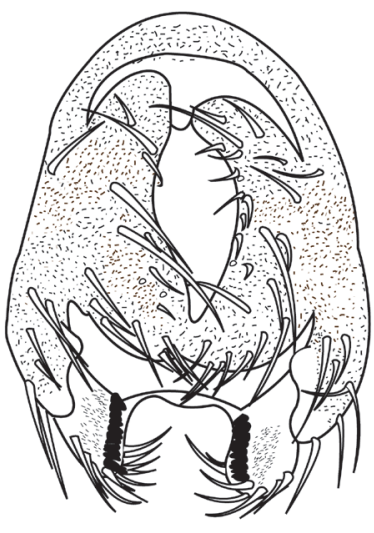

$100 \mu \mathrm{m}$
7

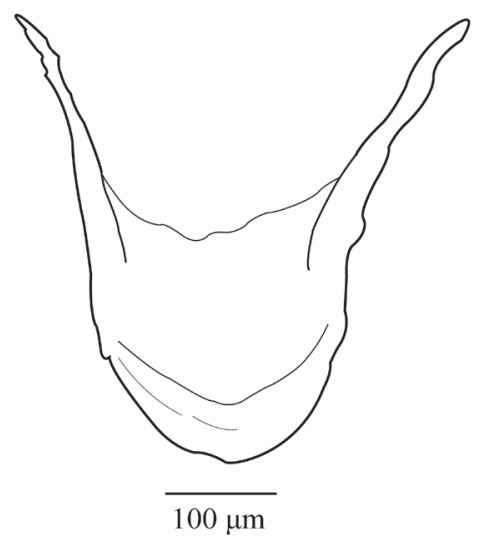

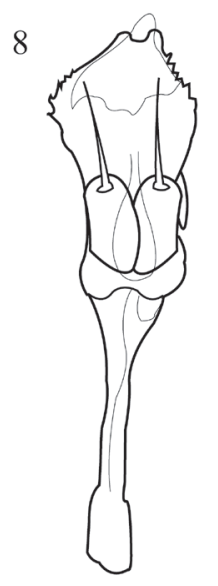

11

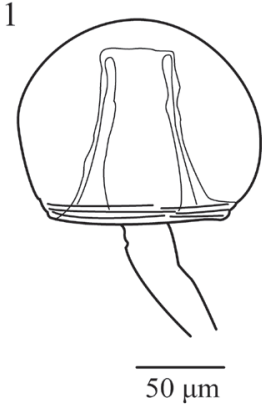

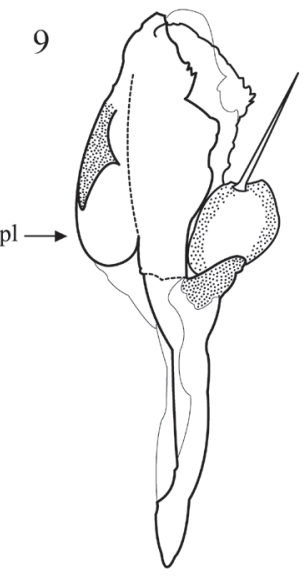

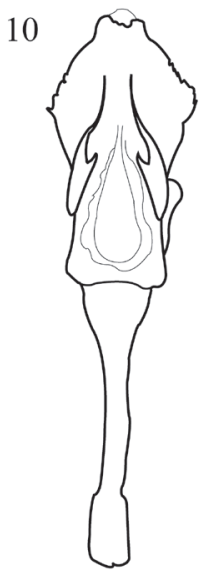

$100 \mu \mathrm{m}$

12

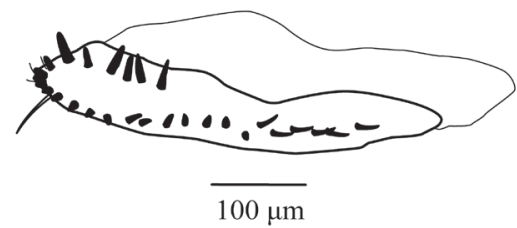

Figs 6-12. Drosophila (D.) yuragyacum sp. nov., holotipo đิ. 6, placa anal; 7, hipandrio; edeago: 8, vista ventral; 9, vista lateral; 10, vista dorsal. Alotipo + : 11, espermateca; 12, ovipositor (pl, proyección laminar).

anteriores. En la parte central y a lo largo de todo el tórax se encuentra una franja delgada ligeramente más oscura que ocupa dos hileras de pelos acrosticales. Escutelo del mismo color que el tórax, con cerdas escutelares anteriores divergentes. Cerda esternopleural media es $1 / 3$ de la anterior. Patas de color amarillo, los tres últimos segmentos tarsales ligeramente más oscuros.

Alas. Color amarillas con la vena transversal posterior ligeramente más oscura. La vena costal III presenta ocho cerdas gruesas. Longitud del ala 4,8 $\mathrm{mm}$; índices alares: $\mathrm{alar}=2,1 ; \mathrm{C}=4,5 ; \mathrm{ac}=1,14 ; \mathrm{hb}=0,25$; $4 \mathrm{c}=0,42 ; 4 \mathrm{v}=1,28 ; 5 \mathrm{x}=1,54 ; \mathrm{M}=0.44$ y Prox $\mathrm{x}=0,47$.

Abdomen. Color amarillo polinoso, sin línea media dorsal; primer y segundo tergito amarillos y ligeramente más oscuros en la parte media; tercer y cuarto tergito más oscuros en la parte media con un diseño semejante a un reloj de arena; quinto y sexto tergito amarillos sin diseño. Además la parte inferior de cada tergito presenta una línea delgada oscura. Testículos de color amarillo.

Genitalia externa. Placa anal micropubescente con cerdas largas y parcialmente unida al epandrio (arco genital). Epandrio micropubescente; parte baja del sin microproyecciones, quitinizada y en punta, con 20 cerdas en el lado izquierdo y 18 en el derecho, (en otros individuos de 14 a 20 cerdas) (Fig. 13). Surestilos rectangulares y micropubescentes en la cara externa; en el surestilo derecho 10 dientes primarios, con 15 cerdas marginales, en el surestilo izquierdo 11 dientes primarios con 13 cerdas marginales, (en otros individuos de ocho a 12 dientes primarios a veces separados, y de 12 a 19 cerdas marginales) (Fig. 14). 


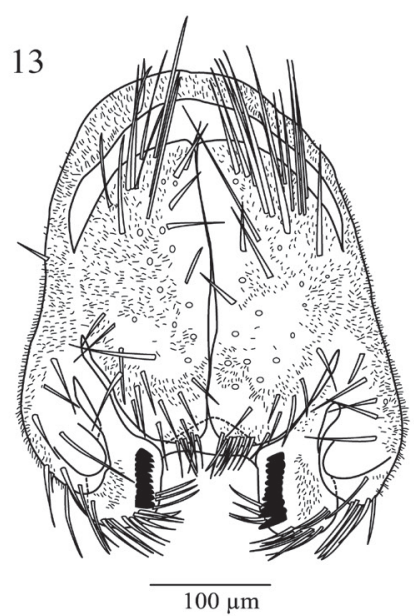

14

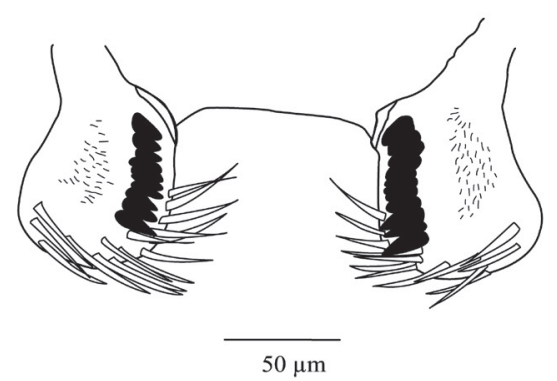

15
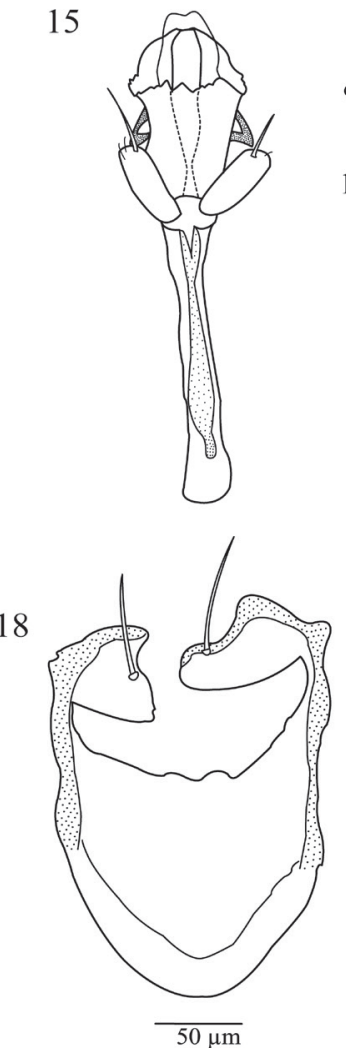

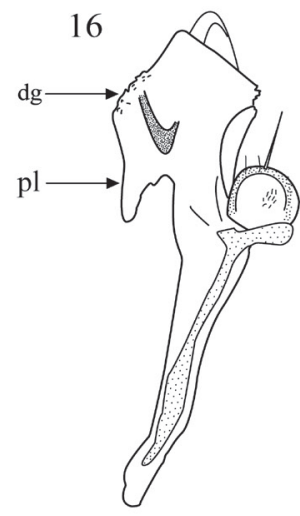

$100 \mu \mathrm{m}$

17

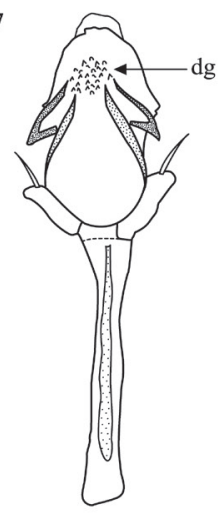

19

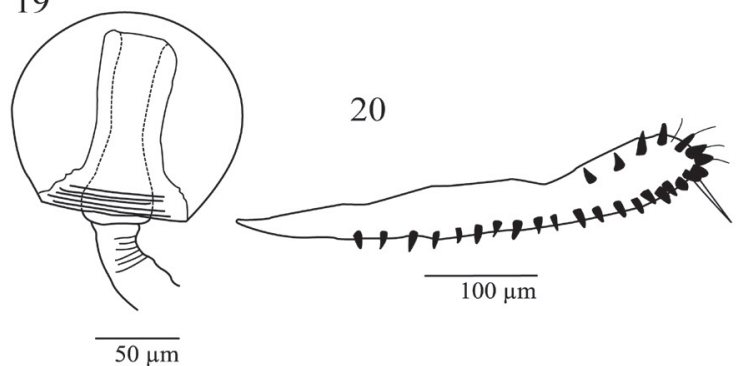

Figs 13-20. Drosophila (D.) yanaurcus sp. nov., holotipo ô. 13, placa anal; 14, surestilo; edeago: 15, vista ventral; 16, vista lateral; 17 , vista dorsal; 18, hipandrio. Alotipo $+:$ 19, espermateca; 20, ovipositor (dg, digitaciones; pl, proyección laminar).

Genitalia interna. Cabeza del edeago quitinizada ancha aserrada lateralmente con la punta ondulada (en otros individuos en punta lisa), con dos proyecciones dorso-laterales como cuernos muy quitinizados; entre estos cuernos se observa digitaciones (Figs 16, 17, dg) y una proyección laminar (Fig. 16, pl) larga, curva y quitinizada hacia la parte anterior. Pie del edeago 1,5 veces más grande que la cabeza; las paráfisis casi circulares con una cerda larga y tres espinas (en otros individuos dos cerdas y de dos a tres espinas), además presenta nueve microproyecciones en la parte ventral. Índice del edeago $=0,67$ (Figs 15-17, 23). Hipandrio del mismo tamaño que el epandrio con borde quitinizado, en forma de escudo y abierto en la parte posterior. Gonopodio pequeño con una cerda larga; arco del hipandrio triangular con una punta membranosa (Fig. 18)

Descripción de la hembra, alotipo y paratipos (individuos muertos en alcohol). Morfología externa. Longitud total (cuerpo + alas) del alotipo $6 \mathrm{~mm}$, longitud total (cuerpo + alas) de los paratipos 6-7,1 $\mathrm{mm}$. Color del cuerpo amarillo polinoso. Igual que el macho pero un poco más oscura.

Alas. Color amarillas con la vena transversal posterior ligeramente más oscura. La vena costal III presenta 11 cerdas gruesas. Longitud del ala $5 \mathrm{~mm}$. Índices 
alares: alar $=2,30 ; \mathrm{C}=5,05 ; \mathrm{ac}=1,12 ; \mathrm{hb}=0,26 ; 4 \mathrm{c}=0,48$; $4 v=1,35 ; 5 x=1 ; M=0,32$ y Prox $x=0,5$.

Genitalia. Ovipositor quitinizado, de color amarillo; en el lado derecho posee cinco dientes discales, 19 cerdas marginales, una espina larga y tres cortas más delgadas; en el lado izquierdo presenta cuatro dientes discales, 19 cerdas marginales, una espina larga y dos cortas más delgadas (otros individuos presentan de cuatro a cinco dientes discales, de 17-27 cerdas marginales, una espina larga y de dos a tres espinas cortas) (Fig. 19). Espermateca pequeña muy quitinizada, redonda con una invaginación que alcanza 3/4 de la espermateca (Figs 20, 25). Índice de la espermateca $=0,7$.

Huevo. Mide aproximadamente $0,7 \mathrm{~mm}$, es ovalado, con cuatro filamentos largos.

Larva. En el último estadio mide aproximadamente $6 \mathrm{~mm}$ y presenta la parte terminal caudal oscura.

Biología. De varias isolíneas fundadas, solo una de ellas dejó descendencia. El ciclo de vida de esta especie es aproximadamente de tres meses: de huevo a larva dura nueve días, de larva a pupa 40 días y de pupa a adulto 45 días. Los adultos viven aproximadamente tres meses (Tab. II). Esta especie fue criada en laboratorio con uvilla (Physalis peruviana), a una temperatura promedio de $8,32^{\circ} \mathrm{C}$ y una humedad relativa promedio de $72,63 \%$.

Etimología. El nombre de la especie hace referencia a la cordillera Yanaurcu de la región del páramo de Papallacta, Pichincha, Ecuador. Palabras quichuas; yana significa negro, yurcu, montaña.

\section{DISCUSIÓN}

Drosophila asiri fue ubicada dentro del grupo D. onychophora (Vela \& RAfael, 2005) debido a las características del edeago. Para incluir a una especie dentro del grupo D. onychophora, es muy importante tomar en cuenta las características del ovipositor de la hembra. El ovipositor del grupo D. onychophora está formado por dos valvas anchas cubiertas de numerosos
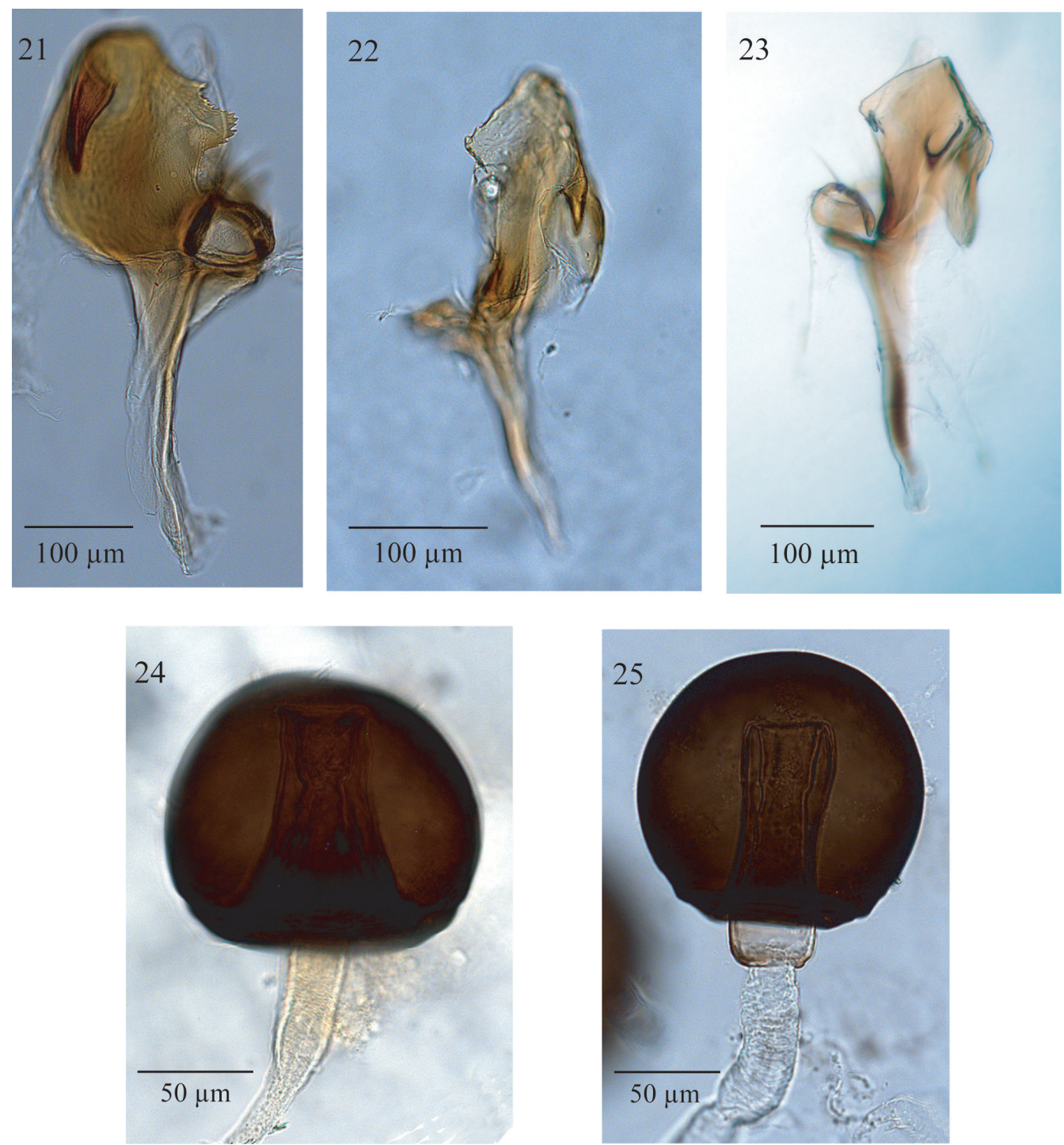

Figs 21-25. Genitalia del nuevo grupo Drosophila (D.) asiri. Edeago, vista lateral: 21, D. (D.) asiri; 22, D. (D.) yuragyacum sp. nov.; 23, D. (D.) yanaurcus sp. nov.; espermateca: 24, D. (D.) yuragyacum sp. nov.; 25, D. (D.) yanaurcus sp. nov. 
dientes pequeños, además son especies con reproducción en flores (VILELA \& BäChLI, 1990). La hembra de D. asiri aún no ha sido encontrada, sin embargo es de suponer que el ovipositor y la espermateca sean similares a los de $D$. yuragyacum sp. nov. y D. yanaurcus sp. nov.

Las características que agrupan a las tres especies a nivel de la morfología externa y la genitalia de los machos y las hembras son varias e identificables; por lo tanto es lícito suponer que las tres especies están emparentadas y que probablemente tienen un ancestro común, aunque presenten características que las diferencian como especies (Tab. I). En adelante proponemos que $D$. asiri ya no pertenezca al grupo $D$. onychophora sino al nuevo grupo de especies $D$.asiri.

Las tres especies del nuevo grupo fueron comparadas con los miembros del grupo $D$. onychophora y también con los miembros del grupo D. macroptera, D. annulimana, D. mesophragmatica y D. bromeliae. El grupo D. macroptera se encuentra distribuido en Centro América, principalmente en México (VILELA \& Bächlı, 2004), mientras que los otros tres grupos son exclusivos de Sudamérica.

Las especies del grupo D. macroptera, particularmente $D$. macroptera, presenta proyecciones laterales en la cabeza del edeago de los machos (VILELA $\&$ Bächli, 2004) como las especies del grupo D. asiri; sin embargo este grupo tiene una distribución muy diferente al grupo $D$. asiri y las especies presentan tamaños pequeños (VILELA \& BäCHLI, 2004) en comparación a las especies del nuevo grupo.

Así mismo, las especies del grupo D. asiri fueron comparadas con las del grupo $D$. annulimana ya que algunas de las especies del grupo son de tamaño grande, miden más de 4 mm (Rocha, 1971; Vilela \& Pereira, 1982; Vilela \& Val, 1983; Pereira \& Vilela, 1987), como las especies del grupo $D$. asiri. Sin embargo la mayoría de los miembros del grupo $D$. annulimana presentan manchas en el tórax (RATCOV \& VILELA, 2007; Tosi et al. 2007) y no tienen las proyecciones laterales en el edeago de los machos, característica muy importante de la genitalia de las especies del nuevo grupo $D$. asiri.

También se comparó a las especies del grupo $D$. asiri con las especies del grupo D. mesophragmatica ya que ambos grupos estarían distribuidos en el mismo rango altitudinal. El grupo $D$. asiri se encuentra distribuido desde los 3200 hasta los $4000 \mathrm{~m}$ de altitud en los andes ecuatorianos, y las especies del grupo $D$. mesophragmatica están distribuidas en América del Sur, principalmente en los países andinos (BRNCIC \& Koref, 1957). Además, en los últimos años en Ecuador, se han encontrado varias especies nuevas del grupo $D$. mesophragmatica viviendo en zonas de altura (2500$4000 \mathrm{~m}$ ) como las especies del grupo D. asiri. Sin embargo las especies del grupo D. mesophragmatica tienen línea media dorsal en el abdomen (BRNCIC \& KOREF, 1957) y los machos presentan en el edeago un proceso dorsal, mientras que los machos del grupo $D$. asiri, no tienen línea media dorsal en el abdomen y presentan en el edeago proyecciones laterales a modo de cuernos.

Por último se comparó al grupo D. asiri con el grupo $D$. bromeliae, puesto que entre los individuos recolectados con $D$. yanaurcus sp. nov. se encontraron grupos de especies asociados a flores (grupo $D$. onychophora y D. flavopilosa). Sin embargo las especies del grupo $D$. bromeliae presentan cerdas prescutelares (Sturtevant, 1916; Val \& Marques, 1996; Silva \& MARTINS, 2004) y no presentan las proyecciones laterales en el edeago.

Se conoce poco sobre la ecología de las drosófilas

Tab. I. Comparación de las características del edeago y distribución ecológica de las especies del grupo Drosophila (Drosophila) asiri.

\begin{tabular}{|c|c|c|c|c|}
\hline & Estructura & D. (D.) asiri & D.(D.) yuracyacum & D. (D.) yanaurcus \\
\hline & cabeza (vista lateral) & forma globosa & forma romboidal & forma romboidal \\
\hline & cuernos & $\begin{array}{c}\text { nacen en la parte más alta de la } \\
\text { cabeza y son largos }\end{array}$ & $\begin{array}{c}\text { nacen en la parte media } \\
\text { doral de la cabeza y son más } \\
\text { cortos que en } D \text {. asiri }\end{array}$ & $\begin{array}{c}\text { nacen en la parte media de la } \\
\text { cabeza y son más cortos que en } \\
\text { D. asiri y que en } \\
\text { D. yuracyacum }\end{array}$ \\
\hline & proyección laminar & ausente & presente, pequeña & presente, grande \\
\hline \multirow[t]{5}{*}{ Edeago } & $\begin{array}{l}\text { parte dorsal de la } \\
\text { cabeza }\end{array}$ & sin digitaciones & sin digitaciones & con digitaciones \\
\hline & abertura dorsal & presente & ausente & ausente \\
\hline & aserrado lateral & irregular y muy pronunciado & $\begin{array}{l}\text { regular y menos } \\
\text { pronunciado }\end{array}$ & irregular y menos pronunciado \\
\hline & paráfisis & $\begin{array}{c}1 \text { cerda larga, } 2 \text { espinas y }+ \text { de } \\
20 \text { microproyecciones }\end{array}$ & $\begin{array}{l}1 \text { cerda larga y sin micro- } \\
\text { proyecciones }\end{array}$ & $\begin{array}{c}1 \text { cerda larga, } 3 \text { espinas y } 9 \\
\text { microproyecciones }\end{array}$ \\
\hline & índice & 0,81 & 1,09 & 0,67 \\
\hline \multicolumn{2}{|c|}{ Localidad (altitud) } & $\begin{array}{l}\text { Volcán Pasochoa } \\
(3260-3310 \mathrm{~m})\end{array}$ & $\begin{array}{l}\text { Quebrada de Cruz Loma } \\
\qquad(3550 \mathrm{~m})\end{array}$ & $\begin{array}{l}\text { Páramo de Papallacta } \\
\qquad(3731-4014 \mathrm{~m})\end{array}$ \\
\hline \multicolumn{2}{|c|}{ Hábitat } & Bosque andino & Bosque alto andino & Páramo, bosque de Polylepis \\
\hline
\end{tabular}


Tab. II. Ciclo de vida de Drosophila (Drosophila) yanaurcus sp. nov. en condiciones de laboratorio; temperatura promedio de $8,32^{\circ} \mathrm{C}$ y humedad relativa promedio de $72,63 \%$ (*, la cópula empezó antes del inicio de la observación, por lo tanto puede durar más de 10 minutos).

Fecha

16 septiembre 2009

28 octubre 2009

F1

9 noviembre 2009

30 noviembre 2009

14 enero -8 febrero 2010

29 enero 2010

3 febrero 2010

2 marzo 2010

5 abril 2010

F2

Observación

fundación de isolínea en tubo con medio de cultivo y uvilla, con hembra colectada en el campo

huevos

larvas

pupas

adultos (8 individuos: $3 \hat{\jmath}, 5$ 웅

observación de cópula durante 10 minutos*

cambio de frasco de los adultos por contaminación con hongos $(2 \hat{\jmath}, 3$ + $)$

huevos

larvas de 1 er y 2 do estadio

larvas de 1er y 2 do estadio

larvas de 3er estadio

pupas (4)

adulto F2

de altura, no existen estudios sobre su ciclo biológico, se desconoce la duración de cada estadio y las estrategias que estarían en juego. En este trabajo se presentan algunos datos sobre el ciclo de vida de D. yanaurcus sp. nov., miembro del nuevo grupo $D$. asiri. Esta especie fue criada en laboratorio a una temperatura promedio de $8,32^{\circ} \mathrm{C}$ y humedad relativa de $72,63 \%$, en medio de cultivo de banano enriquecido con trozos de la fruta de uvilla (Physalis peruviana, familia Solanaceae), simulando las condiciones ambientales del lugar de captura de la especie donde las temperatura promedioes de $4-6^{\circ} \mathrm{C}$ y la humedad relativa es de $90-93 \%$. El ciclo biológico de esta especie dura tres meses y su longevidad también es de tres meses (Tab. II). Los individuos que llegaron al estadio adulto fueron pocos.

Cuando el tamaño de las poblaciones es muy pequeño y fluctúa, existe la posibilidad de que la población se extinga por estocasticidad ambiental o demográfica; este hecho podría ocurrir en poblaciones que se encuentran cerca de los límites fisiológicos (Hoffmann, 2010). Probablemente este sea el caso de las drosófilas de altura que se enfrentan a condiciones climatológicas extremas tales como las bajas temperaturas de los páramos $\left(3^{\circ} \mathrm{C}-8^{\circ} \mathrm{C}\right)$. HoffmanN (2010) demostró que la temperatura afecta la fertilidad y la viabilidad de las drosófilas. Este hecho explicaría en parte la poca descendencia producida por D. yanaurcus sp. nov.

El nuevo grupo $D$. asiri por el momento es endémico de los Andes del Ecuador y se encuentra desde los 3.200 hasta los $4.000 \mathrm{~m}$ de altitud. Estudios futuros de las especies del grupo podrán esclarecer su distribución y ecología, así como su posible asociación a frutos de Solanaceae, ya que esta familia de plantas es abundante en los páramos y bosques andinos del Ecuador.
Agradecimientos. A la Pontifica Universidad Católica del Ecuador por financiar los proyectos: "Caracterización de nuevas especies ecuatorianas de Drosophila" No. E29050 y "Diversidad del género Drosophila en bosques de Polylepis de la Provincia de Pichincha" $N^{o}$. E29181. Al Ministerio de Ambiente por emitir la autorización de investigación científica $\mathrm{N}^{\circ}$. 008-09 IC-FAU-DNB/ MA. Al Dr. Carlos Ribeiro Vilela (Universidad de São Paulo), por sus valiosos comentarios sobre el nuevo grupo D. asiri. Al Dr. Clifford Keil, al M. Sc. Álvaro Barragán y a los revisores anónimos por examinar y comentar este manuscrito. Al personal del laboratorio de Genética Evolutiva, al Dr. Guillermo Ayerve por su ayuda en la latinización de los nombres de las nuevas especies, a las licenciadas Renata León, Gabriela Pozo y Fernanda Salazar (PUCE). A Idea Wild por la donación de materiales.

\section{REFERENCIAS BIBLIOGRÁFICAS}

Bächli, G.; Vilela, C. R.; Escher, S. A. \& Saura, A. 2004. The Drosophilidae (Diptera) of Fennoscadia and Denmark. Fauna Entomologica Scandinavica v. 39. Leiden, Boston Brill. 362p.

Brncic, D. \& Koref-Santibañez, S. 1957. The mesophragmatica group of species of Drosophila. Evolution 11(3):300-310.

Hoffmann, A. A. 2010. Physiological climatic limits in Drosophila: patterns and implications. The Journal of Experimental Biology 213:870-880.

Pereira, M. A. \& Vilela, C. R. 1987. Two new members of the Drosophila annulimana species group (Diptera, Drosophilidae). Revista Brasileira de Entomologia 31(1):13-18.

Rafael, V.; Arcos, G. \& Terán, A. 2000. Ecología y distribución del género Drosophila en Guayllabamba y el Quinche, provincia de Pichincha - Ecuador. Revista de la Pontificia Universidad Católica del Ecuador 65:130-155.

Ratcov, V. \& VileLA, C. R. 2007. A new neotropical species of spot-thoraxed Drosophila (Diptera, Drosophilidae). Revista Brasileira de Entomologia 51(3):305-311.

Rосна, R. 1971. Drosophila brenerae, a new neotropical species of the annulimana group (Diptera, Drosophilidae). Papéis Avulsos de Zoologia 25(16):139-142.

Stlva, A. \& Martins, M. 2004. A new anthophilic species of Drosophila Fallén belonging to the bromeliae group of species (Diptera, Drosophilidae). Revista Brasileira de Zoologia 21(3):435-437.

Sturtevant, A. H. 1916. Notes of north american Drosophilidae with descriptions of twenty-three new species. Annals Entomological Society of America 9(4): 323-343.

Tosi, D.; Pereira, M. A. \& Vilela, C. R. 2007. Polytene chromosomes and phylogenetic relationships in ten Drosophila species of the annulimana group (Diptera, Drosophilidae). Genetics and Molecular Biology 30(4):1169-1180. 
VAL, F. \& MARques, M. 1996. Drosophilidae (Diptera) from the pantanal of Mato Grosso (Brazil), with the description of a new species belonging to the bromeliae group of the genus Drosophila. Papéis Avulsos de Zoologia 39(11):223-230.

Vela, D. \& Rafael, V. 2005. Nuevas especies de Drosophila (Diptera, Drosophilidae) en el bosque Pasochoa, Pichincha-Ecuador. Revista de la Pontificia Universidad Católica del Ecuador 75:69-80.

VilelA, C. R. \& Bächl, G. 1990. Taxonomic studies on Neotropical species of seven genera of Drosophilidae (Diptera). Mitteilungen der Schweizerischen Entomologischen Gesellschaft 63:1-332.
Vilela, C. R. \& Bächli, G. 2004. Revision of the Drosophila macroptera and $D$. rubrifrons species groups, with description of a new Neotropical group (Diptera, Drosophilidae). Mitteilungen der Schweizerischen Entomologischen Gesellschaft 77:1-68.

Vilela, C. R. \& Pereira, M. A. 1982. A new species of the annulimana group of the genus Drosophila (Diptera, Drosophilidae). Revista Brasileira de Entomologia 26(3/4):237-240.

VILELA, C. R. \& VAL, F. C. 1983. Redescription of Drosophila tarsata Schiner, 1868 and description of a new species, both in the annulimana group (Diptera, Drosophilidae). Revista Brasileira de Biologia 43(4):373-380. 